Simple model of laser damage initiation and conditioning in frequency conversion crystals

M.D. Feit, A.M. Rubenchik, J.B. Trenholme

November 8, 2005

Boulder Damage Symposium XXXVII

Boulder, CO, United States

September 19, 2005 through September 21, 2005 
This document was prepared as an account of work sponsored by an agency of the United States Government. Neither the United States Government nor the University of California nor any of their employees, makes any warranty, express or implied, or assumes any legal liability or responsibility for the accuracy, completeness, or usefulness of any information, apparatus, product, or process disclosed, or represents that its use would not infringe privately owned rights. Reference herein to any specific commercial product, process, or service by trade name, trademark, manufacturer, or otherwise, does not necessarily constitute or imply its endorsement, recommendation, or favoring by the United States Government or the University of California. The views and opinions of authors expressed herein do not necessarily state or reflect those of the United States Government or the University of California, and shall not be used for advertising or product endorsement purposes. 


\title{
Simple model of laser damage initiation and conditioning in frequency conversion crystals*
}

\author{
M.D. Feit ${ }^{\dagger}$, A.M. Rubenchik and J.B. Trenholme \\ University of California \\ Lawrence Livermore National Laboratory \\ 7000 East Avenue, mail stop L-463 \\ Livermore, CA 94550
}

\begin{abstract}
Laser conditioning, i.e. pre-exposure to less than damaging laser fluence, has been shown to improve the damage resistance of KDP/DKDP frequency conversion crystals. We have extended our damage model, small absorbing precursors with a distribution of sizes, to describe various damage related properties such as damage density and effects of laser conditioning in crystals. The model assumes the rate limiting process for both initiation and conditioning depends on temperature and that separate threshold temperatures exist for either conditioning or damage initiation to occur. This is reasonable in KDP/DKDP since the melting temperature is far below the temperatures associated with plasma formation and damage events. This model is capable of accounting for some recently observed damageconditioning behaviors.
\end{abstract}

\section{Introduction}

We have previously introduced a simple model ${ }^{\mathrm{i}}$ describing laser damage precursors as small absorbers distributed in size. As noted in that paper, the idea of small absorbers as precursors goes back many years ${ }^{\text {ii iii }}$, but the current model extends these ideas to treat experimentally accessible properties such as damage density and conditioning in a very natural way. This picture has proved successful enough to offer hope that a self consistent model capable of explaining many different experiments may be achievable. The present paper summarizes some recent results using the original simple version of the model while a companion pape ${ }^{\text {iv }}$ describes extensions of the model to accommodate size dependent absorptivity and distributed absorptivity. We also point out several areas of difficulty with this model.

In ref.[i], assuming precursors of fixed absorptivity independent of size and that damage would occur if a certain (unknown) temperature was reached, we derived a simple analytic expression for the fluence necessary for damage $\phi$ as a function of precursor diameter 2a. This relation is shown in Fig.(1). Supposing some underlying distribution of precursors $\mathrm{n}(\mathrm{a})$, then for some test fluence value given by the dashed line in Fig.(1), the precursors with radii between $\mathrm{a}_{\min }$ and $\mathrm{a}_{\max }$ see fluences above their thresholds and contribute to the observed damage density $\rho$, i.e.

$$
\rho(\phi)=\int_{a_{\min }(\phi)}^{a_{\text {may }}(\varphi)} n(a) d a
$$

Here, we have assumed that precursor size is the only selection parameter (in addition to fluence) for damage initiation. If other parameters such as distributions in absorption exist, this expression can be generalized as shown in [iv]. In general, absorptivity can depend on precursor size as pointed out in [1], but the mathematically simplest treatment assumes constant absorption efficiency and this simplest assumption leads to reasonable treatment of a number of experimental results as pointed out above. There are some physical issues with this assumption, however, as mentioned in the summary below. As fluence is increased, damage density $\rho$ changes because the limits of the integral change. Generally, we can expect n(a) to vary roughly as an inverse power of a if there are many more small precursors than large ones and most of the value of the integral in Eq.(1) will come from the lower end. In this case, if $n(a) \sim a^{-(p+1)}$, then $\rho$ will vary with fluence as $\rho(\phi) \sim \phi^{p}$. A number of experiments show $p$ is typically about 3 or larger. Ref.[1] shows how the limiting sizes $a_{\min }$ and $a_{\max }$ depend on fluence and pulseshape. 
This picture can be simply extended to the case of multi-wavelength exposure if the same precursors absorb both wavelengths, perhaps to different amounts. That is by assuming the critical temperature is reached by absorbing light of different wavelengths. In this case, the absorbed fluence which was originally of form $\mathrm{Q} \phi$ with $\mathrm{Q}$ being the absorptivity will now become $\mathrm{Q}_{2} \phi_{2}+\mathrm{Q}_{3} \phi_{3}$ where the subscripts 2,3 refer to two different wavelengths. This implies that the shape of the damage density curve has a universal form in terms of the total absorbed fluence. This effect is illustrated in the model calculations of Fig.(3). Becaise the universal shape of the curve only holds sufficiently far above threshold, the appropriate linear combination of the two fluences can depend on how close to threshold the two fluences are. Additionally, if the absorptivity depends on precursor size, this also leads to the appropriate linear combination depending weakly on the two fluences.

We can describe laser conditioning in this model if we assume that conditioning also depends on heating to a lesser degree than that which causes damage and that there is a conditioning threshold temperature. Possible conditioning mechanisms for which this might be the case are described in ref.[i]. In such a case, there is an additional curve for the fluence necessary for conditioning as a function of precursor size as shown in Fig.(2). When conditioning is carried out at a fluence indicated by the blue (lower) dashed line in Fig.(2), precursors with radii between $\boldsymbol{a}_{\min }^{\boldsymbol{c}}$ and $\boldsymbol{a}_{\max }^{\boldsymbol{c}}$ are made benighn. When a later damage test is carried out at the fluence indicated by the red (upper) dashed line, the damage density that would have occurred without conditioning (Eq.(1)) is reduced by the number of conditioned precursors, i.e.

$$
\rho(\phi)=\int_{a_{\min }(\phi)}^{a_{\max }(\varphi)} n(a) d a-\int_{a_{\min }^{c}\left(\phi_{c}\right)}^{a_{\max }^{c}\left(\phi_{c}\right)} n(a) d a
$$

It is evident from the figure that if the conditioning fluence is high enough, conditioning itself will cause some damage.

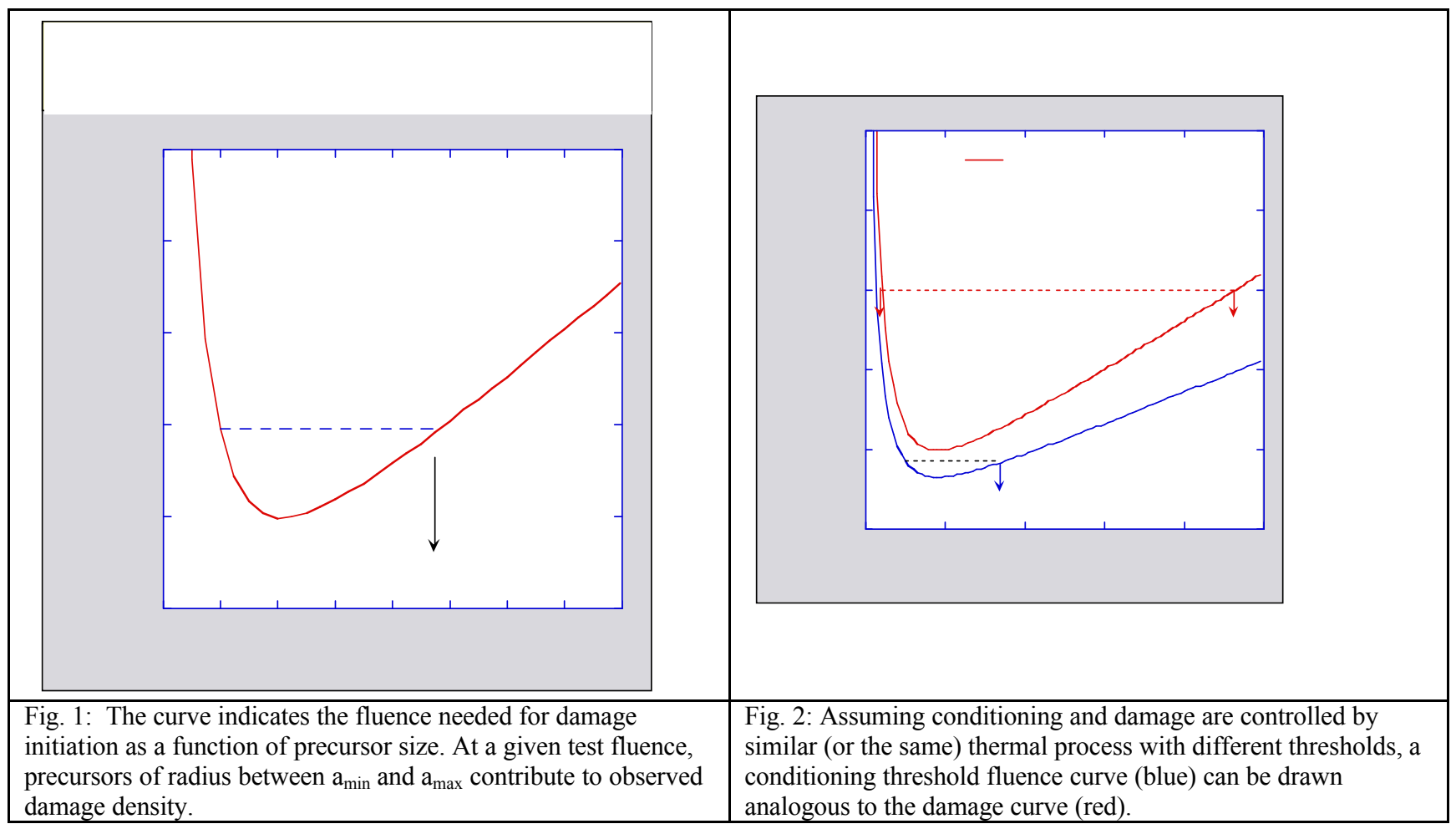




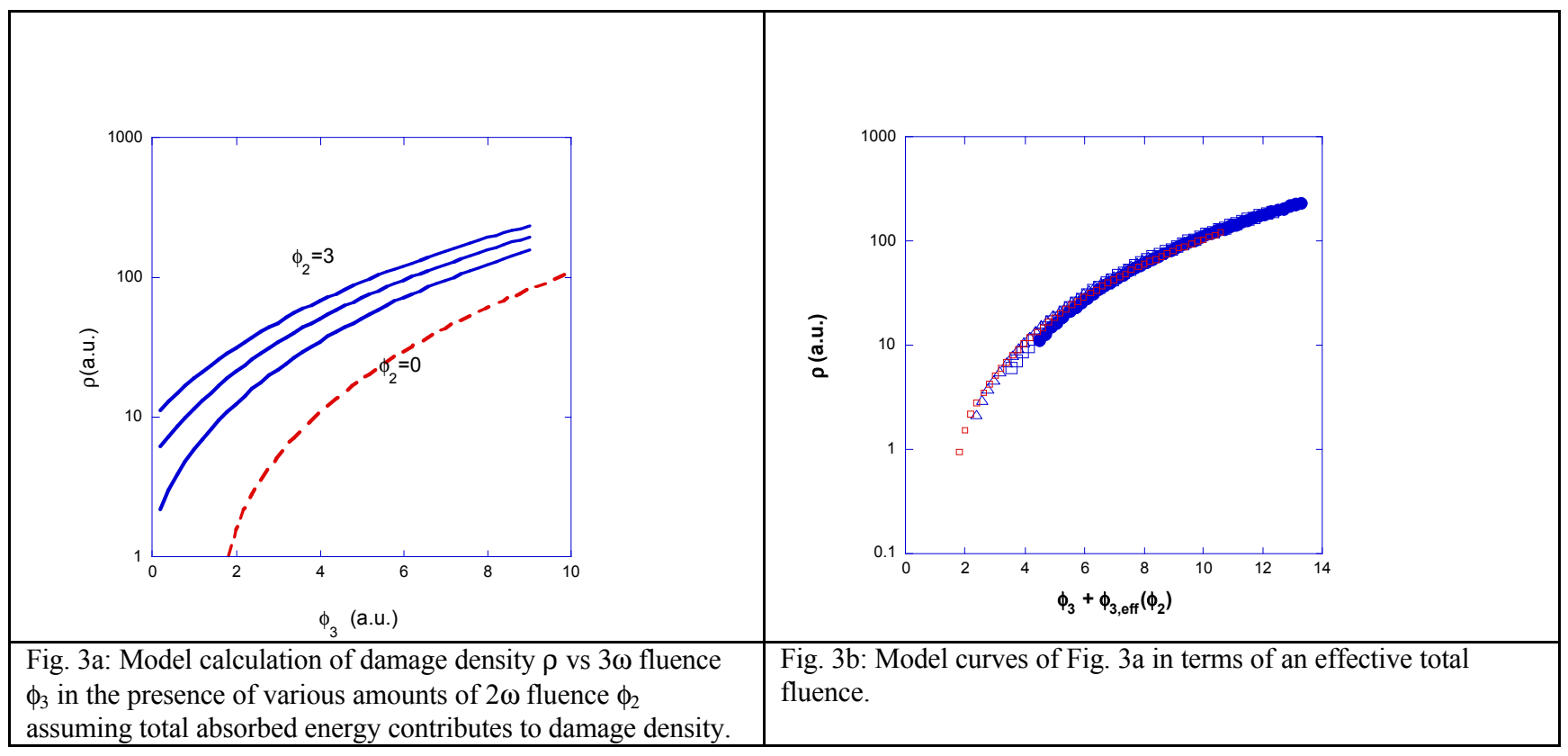

\section{Comparison with experiment}

Laser induced bulk damage densities in DKDP resulting from simultaneous exposure to $2 \omega$ and $3 \omega$ fluences have been measured at Livermore and are presented ${ }^{\mathrm{V}}$ elsewhere in these proceedings. The experimental results from this work shown in Fig. 4 demonstrate the same kind of universal shape of the damage density vs fluence curve shown in the calculated densities of Fig. 3. The theoretical curves assumed the power in $\rho \sim \phi^{p}$ to be $p=3$ which reproduces the observed results reasonably well.
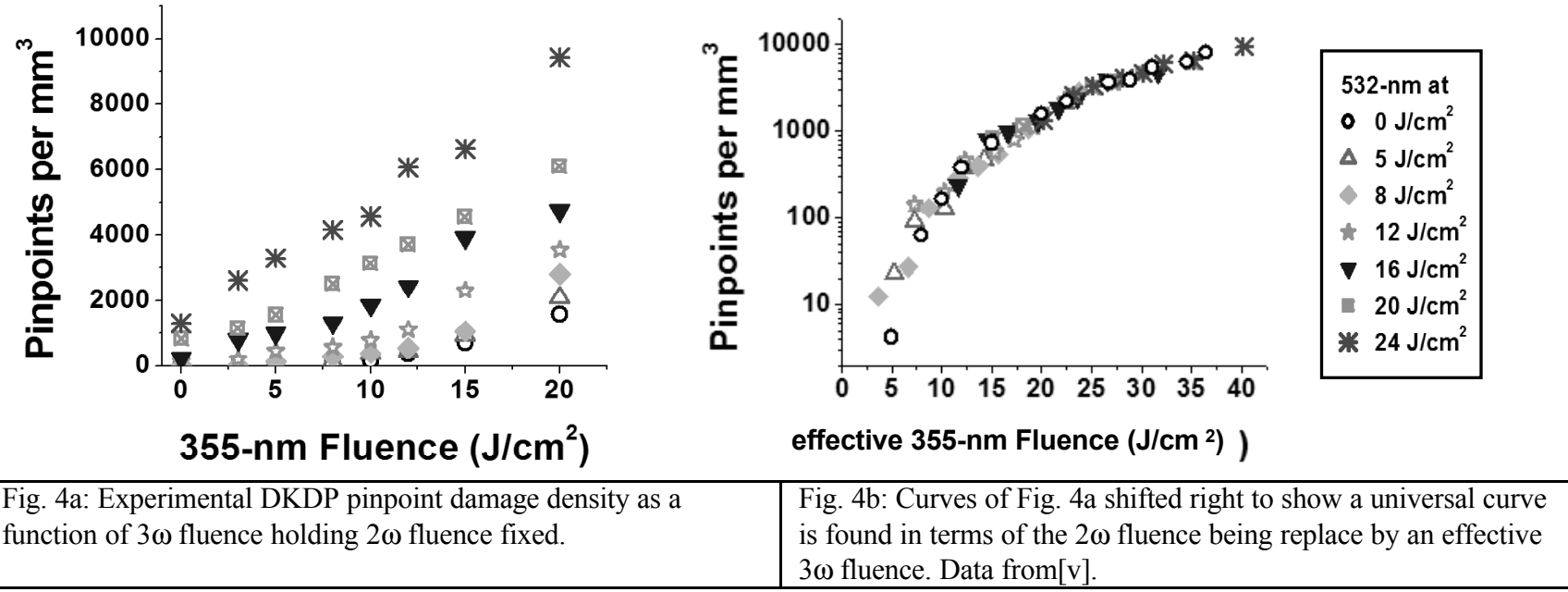

The same group has examined the effect of conditioning with various wavelength pulses on damage density caused by various wavelength pulses and reporte ${ }^{\mathrm{vi}}$ the results at this conference. The dots in Fig. 5 give their results for conditioning and damaging at $3 \mathrm{w}$. For each dataset in the plot, the damage fluence is held fixed (at $15 \mathrm{or} 20 \mathrm{~J} / \mathrm{cm}^{2}$ for the 
two sets of data) and the damage density is plotted as a function of the conditioning fluence. For low values of conditioning fluence, no effect on damage density is found. Eventually, conditioning becomes effective reducing the amount of damage until the conditioning fluence itself is high enough to cause damage. The curves in the figure are fits of the model of Eq.(2). The fit is reasonable as are the values of the few parameters involved (e.g. damage and conditioning thresholds). However, it has proven more difficult to fit the observed $2 \omega$ conditioning $-2 \omega$ damage results to our model. The behavior observed at $2 \omega$ (see[vi]) appears qualitatively different from that seen for $3 \omega$ and $1 \omega$. The ultimate cause of this difference is under investigation.

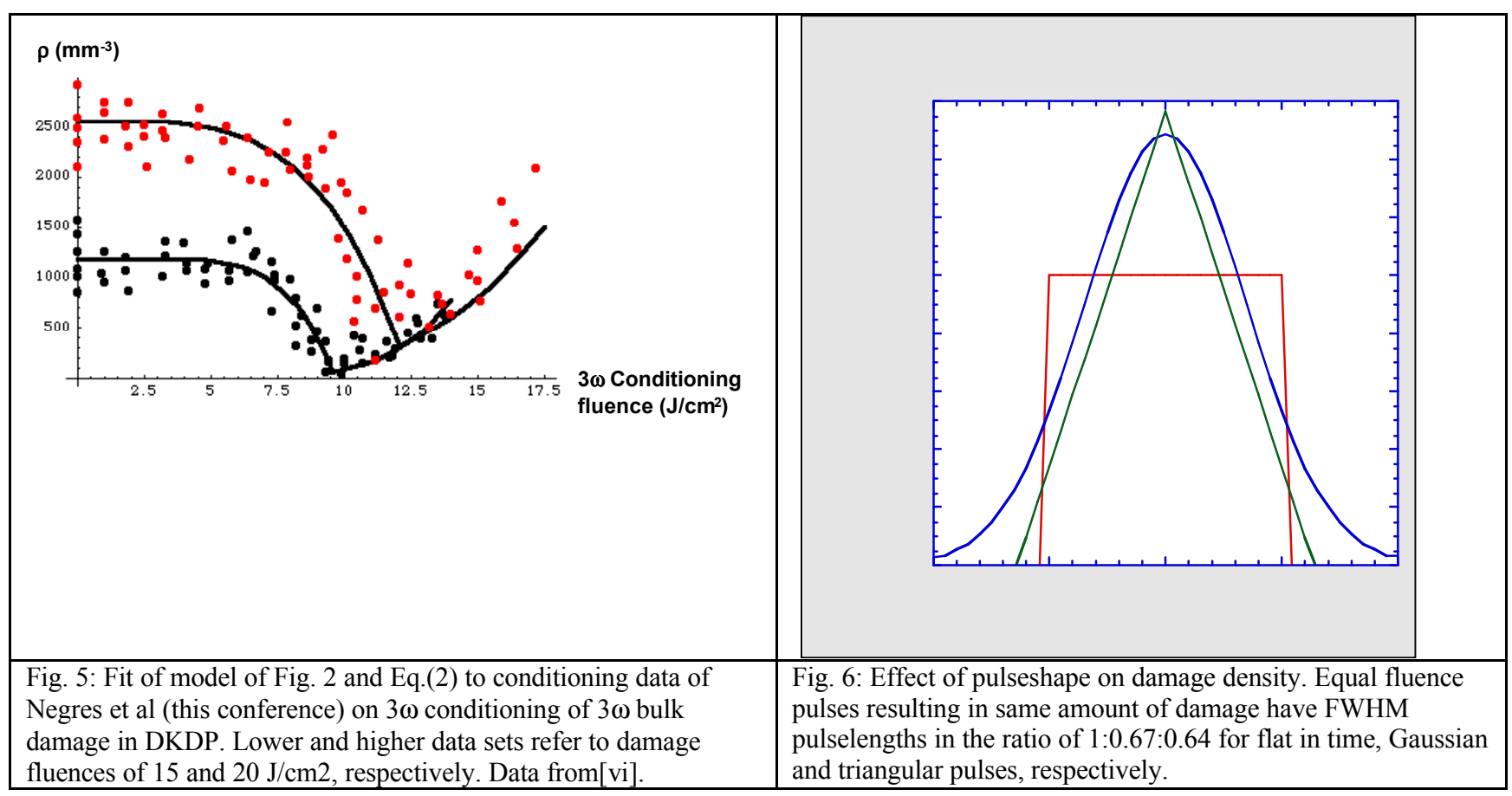

We would like to mention that the formalism presented here allows one to determine not only the effect of pulselength, but also the effect of pulseshape on damage density. Using the formalism of [i], using Eq.(1) where the smallest and largest precursors contributing to damage depends on both fluence and pulseshape, one can find damage equivalent pulses as in Fig.(6). This is important for correlating laboratory results with, say, Gaussian pulses to operational situations where pulseshapes may be different.

Finally, it should be pointed out that the very recent result $\mathrm{t}^{\mathrm{vii}}$ that pulselength dependence of damage density is the same in conditioned and unconditioned KDP is also consistent with the present model.

\section{Summary}

A simple model of bulk damage precursors in KDP/DKDP as a distribution (in size) of small linear absorbers has been proposed. In this model, the balance between energy absorption into and energy loss out of precursors via conduction sets the damage threshold. The model allows a simple theoretical treatment of damage related properties such as damage density, pulselength and pulseshape dependence, multi-wavelength exposure and conditioning in some circumstances. Initial comparisons of experimental results with "reasonable" predictions of the model are encouraging and warrant further model development. However, aside from some minor issues, several more serious issues with the model still remain. The first concerns the nature of the precursors as evidenced by their absorptivity. Comparison with experiment leads to the conclusion that absorption has to be fairly strong and nearly independent of precursor size. Mie theory ${ }^{\text {viii }}$ describes the size dependence of the absorption efficiency of small particles and, in principle, should give a first principles description of the precursors if we knew their composition (need complex refractive index). Thus far, this has been difficult to do self-consistently. Initial attempts to include the size dependence of absorptivity is described in [iv]. 
Secondly, as noted above, it has been difficult to understand some of the conditioning results in terms of this model. Thirdly, measurement ${ }^{\mathrm{ix}}$ of the wavelength dependence of the damage threshold in KDP seems to indicate a multi-photon or at least multi-step absorption process is involved which is difficult to include in the present model.

Thus, this model appears hopeful, but there are still some mysteries to clear up before we can have full confidence in it.

* This work was performed under the auspices of the U.S. Department of Energy by University of California Lawrence Livermore National Laboratory under contract No. W-7405-Eng-48

\section{References}

\footnotetext{
${ }^{i}$ M.D. Feit and A.M. Rubenchik, "Implications of nanoabsorber initiators for damage probability curves, pulselength scaling and laser conditioning", Proc. SPIE 5273, 74 (2004)

${ }^{\text {ii }}$ R.W.Hopper, D.P.Uhlmann. "Mechanism of inclusion damage in laser glass.," Appl.Phys.41,4023-4037 (1970)

iii M.R.Lange, J.K.McIver, A.H.Guenther, T.W.Walker, "Pulsed laser induced damage of an optical material with a spherical inclusion”, Damage in Laser Materials 1982, Nat. Bur. Stand. Spec. Pub. 669, 380-6, (1984)

iv J.B. Trenholme, M.D. Feit and A.M. Rubenchik, "Size selection initiation model extended to include shape and random factors", elsewhere in this proceedings

${ }^{v}$ R. A. Negres, P. P. DeMange, H. B. Radousky, S. G. Demos, "Laser-induced damage in DKDP crystals under simultaneous exposure to laser harmonics", elsewhere this proceedings

${ }^{v i}$ P. P. DeMange, R. A. Negres, C. W. Carr, H. B. Radousky, S. G. Demos, "A multi-dimensional investigation of laser conditioning in KDP and DKDP crystals", elsewhere this proceedings

vii J.J. Adams, J.A. Jarboe, T.L. Weiland, J.R. Stanley, W.D. Sell, R.L. Luthi, J.L. Vickers, D.A. Willard, C.W. Carr, M.D. Feit, A.M. Rubenchik, R.P. Hackel, "Wavelength and pulselength dependence of laser conditioning and bulk damage in doubler cut KDP", elsewhere this proceedings

${ }^{\text {viii }}$ H.C. van der Hulst, Light scattering by small particles, John Wiley and Sons, NY (1957)

ix C.W. Carr, H. B. Radousky, and S.G. Demos, "Wavelength Dependence of Laser-Induced Damage:

Determining the Damage Initiation Mechanisms" Phys. Rev. Lett. 91(12), 127402 (19 Sept. 2003)
} 